\title{
Z- PLASTY: AN EFFECTIVE TECHNIQUE FOR PILONIDAL SINUS
}

\author{
KHAN MRK ${ }^{1}$, BEGUM MN $^{2}$, ISLAM $\mathrm{S}^{3}$, ISLAM AFMA ${ }^{4}$, AHMED RU ${ }^{5}$, KHAN MHA ${ }^{6}$
}

\begin{abstract}
:
Introduction: Pilonidal sinus is a commonly encountered problem with multiple surgical options. With Z- plasty, primary closure ensues faster healing while the alteration of local anatomy reduces recurrence. The extremely low recurrence rate following Z-plasty for pilonidal sinus results from the fact that the operation was planned with the true physiology of the disease in mind. It is the deep natal cleft which predisposes to pilonidal sinuses and this architecture is altered with multiple $Z$-plasty.

Materials and methods: A prospective, observational study was conducted into department of Plastic Surgery and burn, Dhaka Medical College and Hospital. Here 18 patients with pilonidal sinus were treated by $Z$ - plasty between 2013 to 2018.

Result: There was only one recurrence during the follow up time. There were no necrosis of flaps and no wound dehiscence.

Conclusion: The Z-plasty technique is an effective procedure for treating pilonidal sinus in terms of both faster healing and lower recurrence rate.
\end{abstract}

Keywords: Pilonidal sinus, Z-plasty.

J Dhaka Med Coll. 2019; 28(2) : 164-171

\section{Introduction:}

Pilonidal sinus is notorious both for recurrence and for recalcitrance to healing after treatment.

Pilonidal sinus "means appertaining to the nest of hairs" originating from Latin word Pilus Hair, Nidus - Nest.Pilonidal sinus most commonly affects the sacrococcygeal area. The incidence of sacrococccygeal pilonidal disease is not accurately known, but it is reported that $0.7 \%$ of adolescents are affected. Others reported an incidence of 26 per 100,000 regardless of age. However, other locations have been reported, named extracoccygeal (atypical) pilonidal sinus. Those places- according to frequency- are umbilicus (90\%), hand (3.9\%), scalp $(1.7 \%)$, perianal region $(1.3 \%)$, intermammary area $(1 \%)$, face $(0.7 \%)$, periareolar $(0.3 \%)$, penis $(0.3 \%)$, clitoris $(0.3 \%)$ and prepuce $(0.3 \%)$. Risk factors for developing pilonidal sinus include a deep natal cleft, obesity, hirsutism, prolonged sitting, bad personal hygiene and family history . It is called as 'jeep disease'.

Regarding the aetiology, congenital theory is obsolete. Exact mechanisms of development are speculative. Evidence that supports the acquired theory of origin of pilonidal sinuses can be summarised as follows:

- Interdigital pilonidal sinus is an occupational disease of hairdressers, the hair within the interdigital cleft or clefts being from the customers. Pilonidal sinuses of the axilla and umbilicus have also been reported.

- The age incidence of the appearance of pilonidal sinus $(82$ per cent occur between

1. Dr. Mohammad Rabiul Karim Khan, Associate Professor of Plastic Surgery, Sheikh Hasina National Institute of Burn and Plastic Surgery, Dhaka, Bangladesh.

2. Dr. Most. Nurunnahar Begum, Assistant Professor of Plastic Surgery, Sheikh Hasina National Institute of Burn and Plastic Surgery, Dhaka, Bangladesh.

3. Dr. Sharmin Islam, Group doctor, Ascent group, Dhaka.

4. Dr. Abu Faisal Md. Ariful Islam, RS, Plastic Surgery, Department of Plastic Surgery \& Burn Unit, Dhaka Medical College \& Hospital.

5. Dr. Rafiq Uddin Ahmed, Associate Professor of Plastic Surgery, Chittagong Medical College hospital, Dhaka, Bangladesh.

6. Dr. Mohammad Hedayet Ali Khan, Associate Professor of Plastic Surgery, Sheikh Hasina National Institute of Burn and Plastic Surgery, Dhaka, Bangladesh.

Correspondence: Dr. Mohammad Rabiul Karim Khan, Associate Professor of Plastic Surgery, Sheikh Hasina National Institute of Burn and Plastic Surgery, Dhaka, Bangladesh. Contact no: +88 01818915086, E-mail: rabiulpapon77@ yahoo.com

Received: 15-07-2020

Revision: 20-08-2020

Accepted: 29-09-2020 
the ages of 20 and 29 years) is at variance with the age of onset of congenital lesions.

- Hair follicles have almost never been demonstrated in the walls of the sinus.

- The hairs projecting from the sinus are dead hairs, with their pointed ends directed towards the blind end of the sinus.

- The disease mostly affects men, in particular hairy men.

- Recurrence is common, even though adequate excision of the track is carried out.

\section{Aetiology:}

The most important factors for the development of pilonidal sinus are the combination of buttock friction and shearing forces in that area allowing shredded hair or broken hair collected there, to drill through the midline skin, or infection in relation to a hair follicle which allows hair to enter the skin by the suction created by the movement of the buttocks, so creating a subcutaneous, chronically infected, midline track. "Cigarette rolling" movements between the buttocks may drill attached hairs through the skin, a process that is favoured by maceration from sebaceous and sweat gland activity and by the presence of enlarged hair follicles which provides a possible point of entry. From this primary sinus, secondary tracks may spread laterally, which may emerge at the skin as granulation tissue- lined, discharging openings.

The natal crease is attached by an inelastic raphe to the sacrum and coccyx, an arrangement which may produce subcutaneous suction forces on separation of the buttocks. Pressure recorded in the subcutaneous tissue is 4 to $7 \mathrm{~mm}$ of $\mathrm{Hg}$ lower than the atmospheric pressure when the buttocks are spread.

\section{Clinical features:}

- The condition is seen much more frequently in men than in women,

- Usually after puberty and before the fourth decade of life, and

- Characteristically seen in dark-haired individuals rather than those with softer blond hair.
- Patients complain of intermittent pain, swelling and discharge at the base of the spine, but little in the way of constitutional symptoms.

- There is often a history of repeated abscesses that have burst spontaneously or which have been incised, usually away from the midline.

- The primary sinus may have single or multiple openings, all of which are strictly in the midline between the level of the sacrococcygeal joint and the tip of the coccyx.

- Usually, but not invariably (when diagnosis may be confused with anal ûstula or hidradenitis suppurativa), the sinus runs cephalad.

- Carcinoma arising in chronic pilonidal disease has been described, but is exceedingly rare.

\section{Z- plasty:}

$Z$-plasty is a procedure that involves the transposition of two interdigitating triangular flaps. The three limbs of the $Z$ must be equal in length, and the amount of length obtained is related to the degree in which the angles of the $Z$ are opened up. Four fundamental functions of Z-Plasty:

- To lengthen a scar along the direction of the common limb of $Z$

- To break up a straight line or change the direction of the common limb

- To move tissues from one area to another

- To obliterate or create a web or cleft

\section{Sequence of events in Z-plasty:}

- The common limb of $Z$ being along the line of contracture is under tension

- Its ends springs apart when the interdigitating flaps are raised and the fibrous tissue bands of the contractures are divided

- $\quad$ Springing apart of the divided contractures results in change in the shape of the parallelogram and the triangular flaps become transposed

- The contralateral diagonal lengthens and the transverse diagonal shortens 


\section{Design of Z-plasty:}

- All the limbs of the $z$ are equal.

- All angles are equal.

- Angles may be 30 degrees to 90 degrees. Increasing the angle increases the percent gain in length.

- Results in 2 equal flaps.

Multiple Z-plasties may be considered as a number of $Z$-plasties in series or multiple flap $Z$-plasties

\section{Advantages of Multiple Z-plasty over Single Z-plasty:}

Multiple Z-plasty requires less lateral undermining. It has all the advantages of less undermining: less blood loss, no necrosis of the apices of the ûaps, and absence of numbness, which is common with single Z-plasty.

The cosmetic effect is more pleasing as the scars do not extend over the buttocks.

It causes less distortion of the natal cleft. The side tracts can be easily excised as one of the limbs of the multiple Z-plasty. In single Z-plasty it would require a wider ellipse to encompass the side tracts, which would later put the wound closure under tension.

\section{Recurrence:}

Recurrent pilonidal sinus is due to persistence of the factors which initiated the original lesion.

Three possibilities account for this disappointment:

- part of the sinus complex has been overlooked at the primary operation;

- new hairs enter the skin or the scar;

- there is persistence of a midline wound caused by shearing forces and scarring; in this situation, revisional surgery may include re-excision followed by wound closure and obliteration of the natal cleft either by myocutaneous rotational buttock ûap or cleft closure (Bascom) or Z plasty.

- Recurrence rate varies widely from $0 \%$ up to $40 \%$ depending on the surgical approach.

- Recurrence may occur up to 20 years postoperative.

\section{Treatment:}

- Conservative treatment: As the natural history of the condition is usually one of regression, in those whose symptoms are relatively minor, simple cleaning out of the tracks and removal of all hair, with regular shaving of the area and strict hygiene, may be recommended.

- Treatment of an acute exacerbation (abscess): If rest, baths, local antiseptic dressings and the administration of a broadspectrum antibiotic fail to bring about resolution, the abscess should be drained through a small longitudinal incision made over the abscess and off the midline, with thorough curettage of granulation tissue and hair. This procedure may or may not be associated with complete resolution

- Surgical treatment of chronic pilonidal disease: Several treatment modalities have been tried for pilonidal disease, including: -shaving, -incision and drainage, -excision with primary closure, -excision with open packing, -excision with marsupialization,

- flap surgery

Options:

- laying open of all tracks with or without marsupialisation,

- the excision of all tracks with or without primary closure, and

- the excision of all tracks and then closure by some other means designed to avoid a midline wound ( $Z$-plasty, Karydakis procedure, Bascom's procedure).

- An ideal operation should be:

-simple

-must not need prolong hospital stay -have a low recurrence rate -should be associated with minimal pain

- decrease patient's time off work

- It is well known that techniques that place the scar away from the midline have the lowest recurrence rates. 
- The best method of eliminating the tendency for hair puncture to take place at the bottom of a soggy natal cleft is to abolish the cleft and the movements and pressure changes that are its consequence.

- This can be achieved by elevating the midline depression with a Z-plasty, which also dispenses with a weak midline scar. Zplasty furthermore provides a method of achieving successful primary wound healing. Z-plasty achieves healing by primary intention, and by modification of the local environment prevents recurrence.

\section{Patients and method:}

- A prospective, observational study design was used.

- Patients were diagnosed clinically and admitted through outpatient department after preoperative assessment.

- Written informed consent was taken and surgery was performed by the same surgical team.

- All patients who presented with pilonidal disease between 2013 to 2018 at $\mathrm{DMCH}$ were included.

\section{Surgical technique:}

- Before operation, a low-fibre diet is begun, and the patient is encouraged to empty his bowels.

- Under SAB or endotracheal anaesthesia, and with the patient in a moderate jackknife position, the primary sinus is probed to determine its extent, and skin flaps are marked, usually at an angle of 50 to 60 degree, although an angle of 35 to 40 degree has been used without imperiling vitality.

- The natal crease, raphae, primary sinuses, related midline pits and deep granulation tissue with its embedded hairs are then excised in a long ellipse of skin 1 to $2 \mathrm{~cm}$ wide, extending from the broadened triangular area over the upper part of the sacrum down to about $2 \mathrm{~cm}$. from the anal margin, at which point the hair pattern becomes peri-anal. Residual granulation tissue and secondary sinuses are curetted or excised and sutured. Skin hooks are applied at either ends of the wound, and traction is applied.

- Appropriate markings for Z-plasty are made, with an optimum angle of 45to 60 degrees, taking into consideration the principles of plastic surgery.

- The number of cuts depends on the length of the wound and usually varies from 2 to 5 .

- The cuts are deepened to the subcutaneous fat, and the ûaps are undermined and mobilized. Extensive undermining is avoided to prevent the risk of devitalization of the flaps. The flaps are then suitably inter digitated.

- The sub cuticular tissue is sutured and the skin is sutured with ûne interrupted sutures.

- The apex of the ûaps is sutured by partly subcuticular mattress sutures to prevent necrosis. Skin closure is with simple everting sutures.

- Insertion of a negative suction drain.

- A staged procedure has been used with pilonidal abscess . After the ellipse of skin has been removed, pus is evacuated and sent for culture, the cavity is packed with gauze soaked with povisep. Three to five days later, the patient is returned to the operating theatre and Z-plasty carried out.

\section{Design and Marking:}

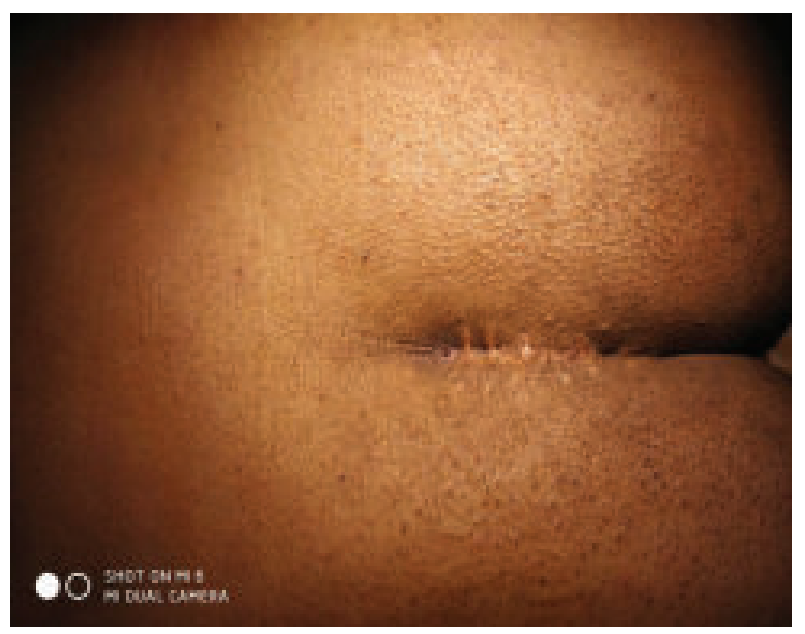

Fig.-1: Chronic pilonidal sinus 

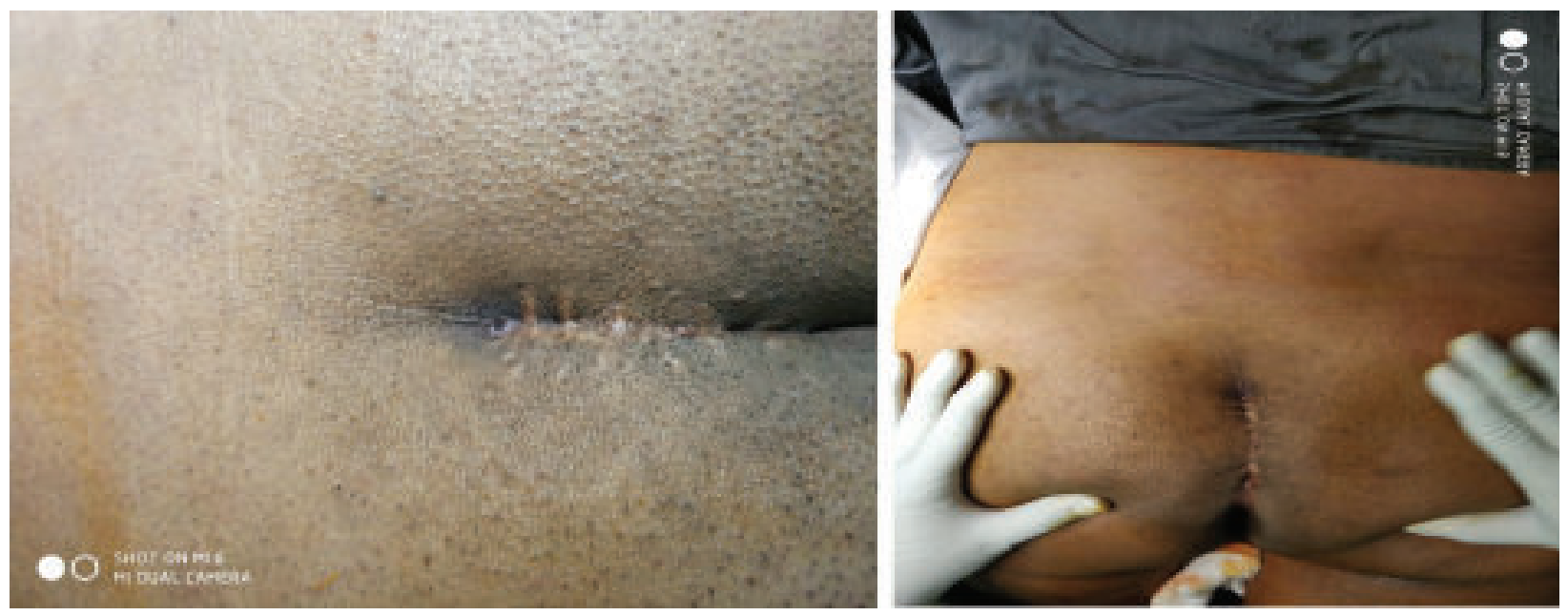

Fig.-2: Chronic pilonidal sinus

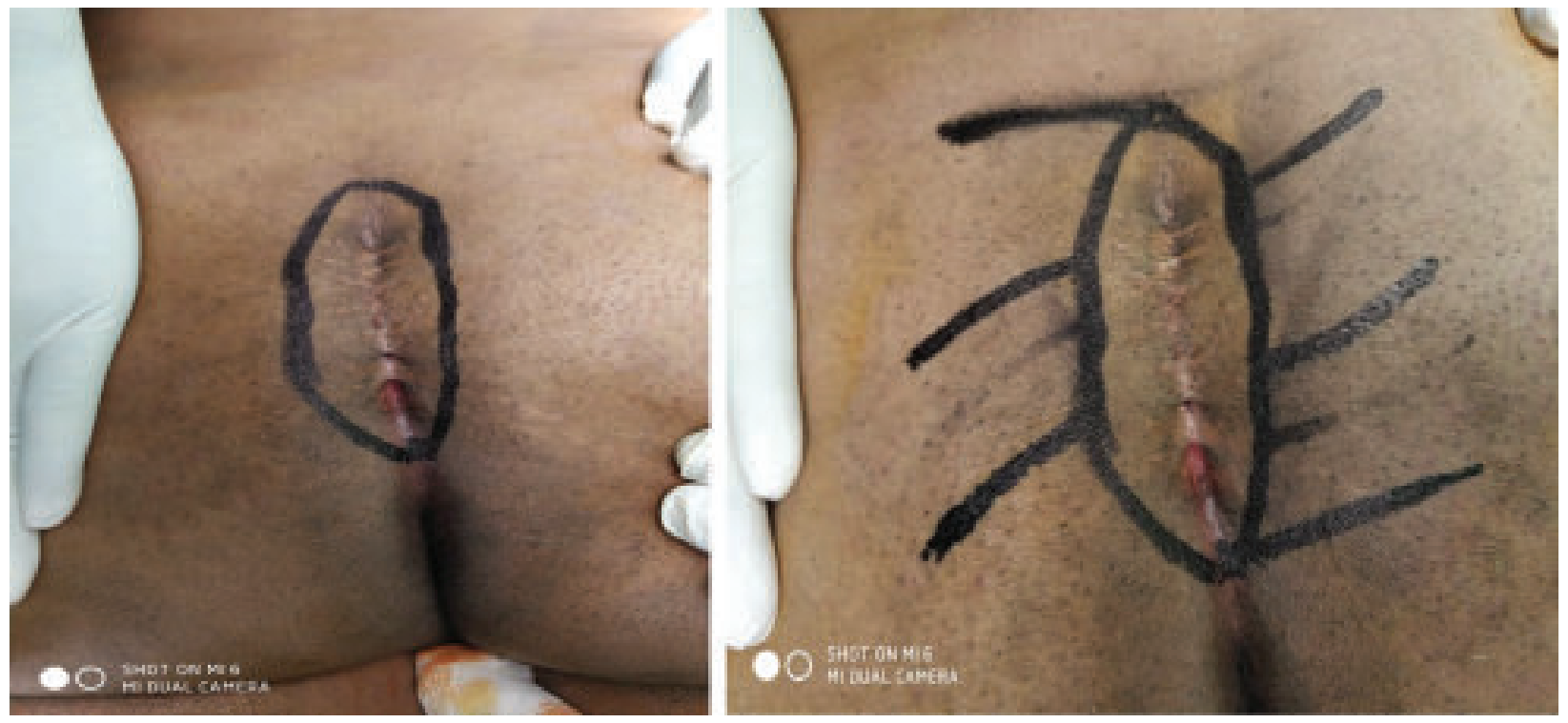

Fig.-3: Multiple $Z$ plasty marking done

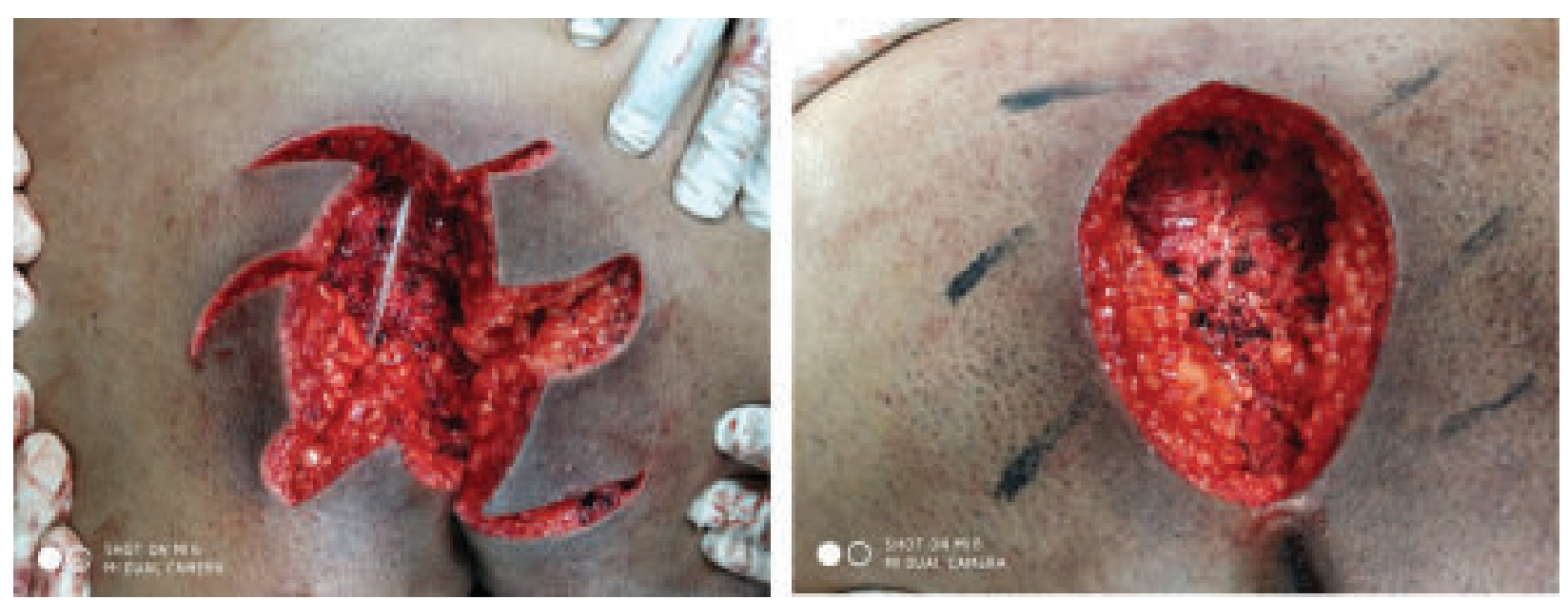

Fig.-4: Multiple $Z$ plasty cut taken 

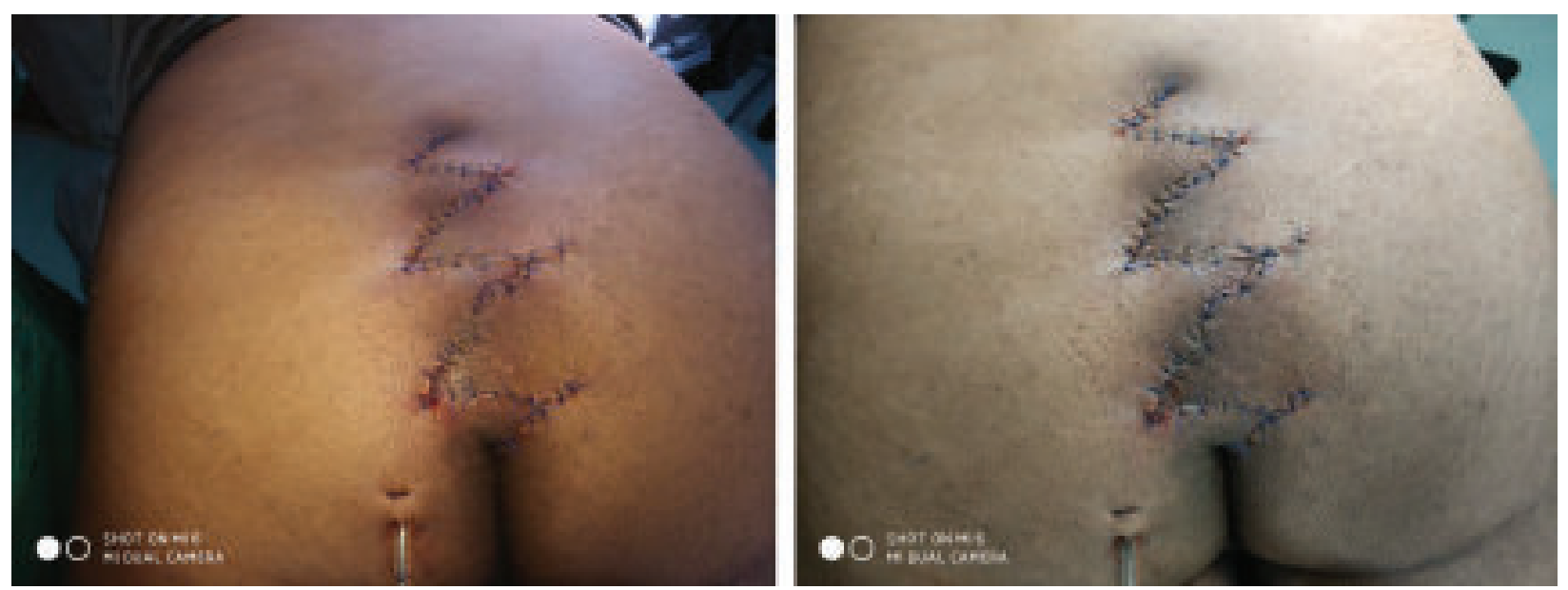

Fig.-5 : Flap interdigitate
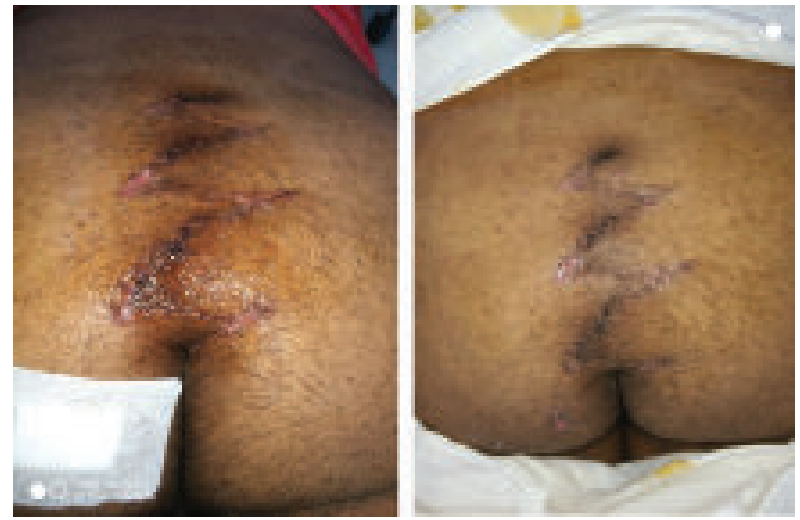

Fig.-6: Sutures removed

\section{Postoperative care:}

All patients were put on a standard postoperative regime that included:

- Antibiotic according to wound swab culture and sensitivity.

- After the operation, adequate analgesia ensured and as the patient is nursed in the lateral posture or prone and is taking the low-fiber diet.

- Maintaining nutrition, adequate hydration, oxygenation and correction of anemia to expedite wound healing process.

- Sequential clinical examinations for arterial insufficiency and venous congestion.

- Check dressing was done on $5^{\text {th }}$ postoperative day.

- To assess viability of the flaps, they were monitored for color, local temperature, tissue turgor, dermal bleeding, flap congestion, blister formation, edema, hematoma, seroma, marginal necrosis and wound infection. Dressing of skin donor site would be monitored for soaking or bad odor. For any sign of infection from any of operative area, wound swab was taken and sent for culture and sensitivity.

- Drainage is ceased when less than $10 \mathrm{ml}$ of serous liquid are discharged in 24 hours.

- The patient is allowed up on the seventh day, and discharged from hospital after removal of the sutures between the 10 to 14 days.

- Shaving of the area is advised for three months until maturation of the scar has occurred.

- Return to full activity is possible the week after the patient's discharge from hospital.

\section{Follow up of the patients:}

Patients were advised to come for follow up on 1,3 and 6 month post operatively for assessment of outcome of the procedure and to identify any recurrence

\section{Result and Observation:}

Reconstructive surgery is always a magnificent combination of classical principles of surgical technique, critical anatomical assessment and application with innovative formulation of newer options. Pilonidal sinus is notorious both for recurrence and for recalcitrance to healing after treatment. Z-plasty is a good option for pilonidal sinus. 
- 18 patients, including three with recurrence following other procedures, have been treated by $Z$-plasty.

- There were 12 (66\%) males and 6 (33\%) females.

- Majority were in the age group of $21-30$ years $(74 \%)$ which is a considerable loss to society due to loss of man working hours.

- The operating time ranged between 60-90 minutes .

- Mean postoperative stay was 7 days and return to work was between 14 to 18 days.

- One patient (5\%) developed recurrence.

- Our study advocates that $z$-plasty technique provides a reasonable method of treatment of sacrococcygeal pilonidal sinus that flattens the natal cleft with low overall incidence of complications and a low recurrence rate and the advantage of less frequent dressings, short hospital stay and rapid return to work.

\section{Discussion:}

Sacrococcygeal pilonidal disease occurs in the midline. Increased depth of the intergluteal sulcus leads to an anaerobic media and increased anaerobic bacterial content. Although many surgical and non-surgical treatments have been described, the ideal treatment method has not yet been established for pilonidal disease.

- There are 2 main surgical options; open method and closed method. Excision of the pilonidal sinus and leaving the wound open to heal by secondary intention has the disadvantage of prolonged healing time with frequent painful dressings and delayed return to work. However, it provides a simple easy way with low recurrence rates. In closed method the wound is closed either primarily or with flap. Primary wound closure is a simple rapid technique with rapid recovery and return to work with less frequent dressings but with high recurrence rates due to placing the suture line in the midline with high tension. Flap- based treatment helps to flatten the natal cleft decreasing hair accumulation, friction and maceration. It also provides a tension free closure with the suture line placed off the midline. Different local flaps could be used. $Z$-plasty is preferred when the pathology is limited to the midline. Z-plasty provides a tension free technique with decreased recurrence by decreasing risk factors by flattening the natal cleft, decreasing hair accumulation, decreasing friction and skin maceration, decreasing vacuuming effect of the natal cleft and placing the suture line off the midline .

- The extremely low complication rate following $Z$ - plasty for pilonidal sinus results from the fact that operation was planned with true pathophysiology of the disease in mind, that it is the deep intergluteal fold which predisposes to pilonidal sinuses and this architecture is materially altered with $Z$-plasty. In pilonidal disease, the Z-plasty eliminates the deep natal cleft by bringing healthy, lateral skin and subcutaneous tissue into the midline. Excision and $\mathrm{Z}$ plasty together can lead to a low recurrence rate with rapid healing. $Z$-plasty excises the inflamed area as well as converts the deep natal cleft into a plateau. It is known that pilonidal sinus does not occur on a flat or a convex surface. Also the directions of the hairs are altered away from the midline. It leaves no midline scar, largely prevents maceration, reduces suction effects in the soft tissues of the buttocks, and minimizes friction between two adjacent surfaces.

- Z-plasty excises the inflamed area as well as converts the deep natal cleft into a plateau. It is known that pilonidal sinus does not occur on a flat or a convex surface. Also the directions of the hairs are altered away from the midline. It leaves no midline scar, largely prevents maceration, reduces suction effects in the soft tissues of the buttocks, and minimizes friction between two adjacent surfaces.

- The environmental factors responsible for pilonidal sinus and its recurrence are eliminated. Z-plasty modifies the local environment so as to counter those mechanisms which promote recurrence, and furthermore provides conditions 
suitable for wound healing by primary intention.

- Excision of pilonidal sinus followed by primary closure with Z-plasty technique had the advantage of early wound healing, short postoperative stay and low risk of recurrence.

- In short, the procedure of Z-plasty : prevents accumulation of debris and maceration leaves no residual pits, which might be the starting point for penetration of hairs elevates the contours of the natal cleft to a level nearer that of buttocks eliminates the ûbrous raphae reduces the effects of both differential friction between the buttocks externally and differential movement and suction subcutaneously.

- The control of recurrence, it is believed, is the consequence of proper local environmental control ; narrow elliptical excision removes all other midline pits and crypts which could prove starting points for further hair drilling or aspiration of foreign material ; the midline contour is elevated, and, as a result, hairs and debris cannot collect in a deep cleft liable to minor sepsis and maceration ; a reduction of differential friction between opposing buttock surfaces is obtained; in addition, $\mathrm{Z}$-plasty avoids a midline scar with impaired resistance to the drilling of hairs, and eliminates subcutaneous suction forces still operative in midline scars.

\section{Conclusion:}

The Z-plasty technique is an effective procedure for treating pilonidal sinus in terms of both faster healing and lower recurrence rate.

\section{References:}

1. Steele SR, Perry WB, Mills S, Buie WD. Practice parameters for the management of pilonidal disease.2013;56:1021-1027.[PubMED]

2. Ommer A. Berg E. breikopf C, et al. S3- Leitlinie: Sinus Pilonidalis. Coloproctology. 2014;36: 272-322.

3. Plewig G, Kligman AM. Berlin Springer- Verlag: 1975. Acnae Morphogenesis and treatment; pp. 192-193.

4. Bascom J. Pilonidal Disease: origin from follicles of hairs and results of follicle removal as treatment . Surgery. 1980;87:567-572.

5. Karydakis GE Easy and successful treatment of pilonidal sinus after explanation of itcausative process. Aust N Z J Surg. 1992;62:385-389.

6. Fazeli MS, Adel MG, Lebaschi AH. Comparison of outcomes in $Z$ - plasty and delayed healing by secondary intention of wound after excision of sacral pilonidal sinus: results of a randomized, clinical trial. Dis Colon Rectum. 2006;49(12):1831-1836. 